\title{
Use of community forums to increase knowledge of HPV and cervical cancer in African American communities
}

\author{
Dede Kossiwa Teteh $^{1}$ (1) - Lenna Dawkins-Moultin ${ }^{1}$. Chartay Robinson ${ }^{2} \cdot$ Victor LaGroon $^{1} \cdot$ Stanley Hooker $^{1}$. \\ Kenneth Alexander ${ }^{3} \cdot$ Rick A. Kittles ${ }^{1}$
}

Published online: 15 April 2019

○) Springer Science+Business Media, LLC, part of Springer Nature 2019

\begin{abstract}
Cervical cancer adversely impacts African American communities. While disparities in incidence remain unclear, communities continue to use forums to increase cervical cancer education. The purpose of this paper is to examine the efficacy of using community forums to increase human papillomavirus vaccine (HPVV) and cervical cancer knowledge in African American communities. This study is a one-group pretest-posttest study design using a 17 -item questionnaire to collect data from 412 participants in diverse communities. Our analyses revealed perceived knowledge increased significantly after the forums for African American participants. For African Americans, perceived knowledge prior to the forums was explained by gender, access to care, and trust in clinical trials. After the forum, perceived knowledge was associated with access to care and trust in vaccines. Participants who had health insurance reported higher perceived HPV and cervical cancer knowledge and greater trust in vaccines. This study found community forums that address the cultural and historical context of research mistreatment related to HPVV development and include diverse racial/ethnic representation of stakeholders may be a useful strategy to increase HPVV, and cervical cancer knowledge in African American communities.
\end{abstract}

Keywords Community forums $\cdot$ Cervical cancer $\cdot$ HPV $\cdot$ Screening $\cdot$ Knowledge $\cdot$ HPV vaccine

\section{Introduction}

Human papillomavirus (HPV) is a common sexually transmitted infection that affects almost 79 million Americans yearly [1]. Most HPV infections are temporary and are usually cleared by our immune system. Infections that are not cleared could lead to cancers of the cervix, vulva, penis or anus. Cervical cancer is the most common HPV-associated cancer for women. Approximately $70-80 \%$ of cervical cancer cases are caused by HPV types 16 and 18 [2]. In the United States in 2017, there were approximately 12,000 cases of cervical cancer each year, and 4200 cervical cancer

Dede Kossiwa Teteh

dteteh@coh.org

1 Division of Health Equities, Department of Population Sciences, City of Hope Medical Center, Duarte, CA 91010, USA

2 Institute of Translational Medicine, University of Chicago, Chicago, IL 60637, USA

3 Nemours Children's Health System, Orlando, FL 32827 , USA deaths [3]. Fortunately, the HPV vaccine has been found to be effective in preventing cervical cancer.

In the past 40 years, death rates due to cervical cancer have decreased more than $50 \%$; primarily due to increased screening and better treatment [4]. However, the rates of cervical cancer diagnosis vary across race and ethnicity [5]. In 2014, Hispanic and Black women carried the greatest burden of cervical cancer diagnosis. Black women were, however, more likely to die from the disease than other race and ethnic groups. Some researchers have offered partial explanations for disparities between Black and White women, including factors such as race [6], socioeconomic status [7], access to care [8], and stage of disease and treatment type [9]. However, other researchers have not found these factors to be associated with why Black women are more likely to die from cervical cancer than their White and Hispanic counterparts $[10,11]$.

Intervention efforts to increase human papillomavirus vaccine (HPVV) uptake and reduce cervical cancer have focused primarily on individual behavior and provider/ practice change [12-14]. Results from these studies suggest provider recommendation and vaccine availability and 
accessibility may be the most effective strategies to increase uptake $[14,15]$. Interventions, however, have not had the same effectiveness across populations, and there remain persistent racial disparities in HPVV knowledge and uptake. Blacks and Hispanics have greater knowledge deficiencies than non-Hispanic Whites [16] and both groups have reported mistrust of the vaccine and a need for more information about its safety and efficacy [17]. Blacks are also $10 \%$ less likely than whites to have received an HPV vaccine [18], are less likely to get an HPV vaccination recommendation from providers [19], are less likely to complete the series, and have the highest rate of HPV prevalence [20].

Despite the racial disparities, there is a dearth of research that has attempted to elucidate strategies that may be more efficacious based on cultural background or may be better suited for specific populations. A scan of literature reviews completed between 2013 and 2018 [13, 14, 19, 21, 22] revealed no study that measured intervention outcomes or strategies related to culture or ethnic differences, even though orientations to medicine and health are associated with culture [23, 24]. It may be useful, therefore, to explore interventions for HPVV through sociocultural lens that consider issues such as familial and communal relationships and influences. This is particularly important since individual and familial factors are the main determinants of HPVV completion [14].

Community forums have been identified by various scholars as a useful community engagement tool to increase knowledge of health issues, engage in dialogue with community members, and develop community-based participatory interventions and research designs [25-28]. For example, to increase stroke awareness and gather community perspectives about prevention and research participation, Bharmal et al. [25] employed community-partnered participatory research principles. Their forum panelists included clinicians, researchers, caregivers and survivors. To analyze the regional differences in awareness and attitudes on genetic ancestry testing, Jonassaint and colleagues [28] used community forums to respond to questions from the community about testing, genetics and disease. Similarly, Davis and colleagues [26] used an Audience Response System to obtain relevant feedback on cancer education initiatives and engage community members. The use of forums to engage communities is also well documented in cancer prevention efforts $[27,29,30]$.

Community outreach and engagement efforts focus on addressing a need presented by the community, using community informed approaches to address the need, and applying engagement and outreach strategies that bring people of respective communities together [29, 31]. Meade and colleagues [30] assert, "outreach programs are important tools for bringing cancer education and screening services directly to community members and serve to contribute to reducing health disparities" (p. 71). Furthermore, the use of a community-centered approach to cancer education allows for the inclusion of a socio-cultural context for improving community health, first through awareness and then through implementation of concerted prevention initiatives.

Despite the potential benefits of community forums, it is underutilized in HPVV interventions. Our search of the scholarly literature found no study that tested the efficacy of community forums as a strategy to increase HPVV knowledge and acceptability. But community forums may be particularly useful among Blacks and Hispanics because it leverages the collective orientation of these communities and builds trust [27, 32]. In composing community forum panels for minority communities, it may be useful to include representation from the pharmaceutical industry to respond to concerns about vaccine safety and efficacy. Historically, the pharmaceutical industry's involvement in the uptake of HPVV revolved primarily around marketing and influencing policies and legislations [33]. Giving communities a chance, however, to talk directly with the makers of the vaccine and have their questions answered may help to allay mistrust and increase knowledge, acceptability, and uptake.

The purpose of this paper is to examine the efficacy of using community forums to increase HPVV and cervical cancer knowledge in African American communities.

\section{Methods}

\section{Study Design}

We used a one-group pretest-posttest study design to assess change in knowledge of HPV and cervical cancer in diverse communities before and after a series of community forums. Pre and posttest assessments were used to measure two sets of cross-sectional data collected on the same population. The forums were convened by Community Engagement Centering on Solutions (CECOS) - the community outreach branch of the University of Chicago Cancer Research Center (UCCRC) - as part of an HPV outreach initiative. The initiative Our daughters, our duty: The facts about HPV and cancer-was a response to the disproportionately higher rates of cervical cancer among Blacks and Hispanics in Illinois, at the time [34]. A community health needs assessment by the UCCRC identified HPV and cervical cancer as two of the most requested topics for health education. Community mistrust of the health care system, and lack of understanding of HPV and the newly developed HPV vaccine, also presented a pronounced need for community education.

Community Engagement Centering on Solutions used a community-centered and driven engagement model to bring clinicians, researchers, community-based organizations, cancer survivors, and pharmaceutical industry representatives 
together with community members to improve knowledge and attitudes towards the HPV vaccine and cervical cancer prevention. The panel was composed of an African American academician who discussed medical mistrust in minority communities and mistrust of HPV vaccine; a representative from the pharmaceutical industry who talked about HPVV development process, safety and efficacy; a community advocate who spoke about the importance of HPVV; and shared her experience with receiving the HPV vaccine. Forum activities were supported by two full-time staff members and four volunteers.

The goal of these forums was to create an interactive and informative bi-directional dialogue between the community and panelists about HPV, cervical cancer and the viability of the HPV vaccine as a form of prevention. Data were collected between March 2009 and March 2010 and analyzed using IBM SPSS version 24.

\section{Data Collection}

All community forum participants provided written informed consent. Data collection procedures and forms were approved by the University of Chicago's Institutional Review Board. Data were compiled from responses to a 17-item questionnaire completed by participants who attended the three forums held at Kennedy King College and Daley College.

\section{Measurement}

The questionnaire captured information regarding demographics, perceived and actual knowledge, health practices, and attitudes.

\section{Demographic}

Seven participant demographic items were assessed: how participants heard about the event, participants' community role, household income, highest level of education completed, age range, race and gender. Questions included: (a) What is your age range? Response options ranged from 19 or younger to 60 and older. (b) What is your gender? Response options were male or female.

\section{Effectiveness of Oral Presentations}

The effectiveness of the oral presentations was assessed using a Likert scale ranging from poor to excellent (1 to 5) for each speaker.

\section{Perceived Knowledge}

Perceived knowledge was examined using a Likert scale ranging from poor to excellent (1 to 5). The following questions were used to assess perceived knowledge prior to and after the forum presentations: (a) Prior to this event how would you rate your understanding of HPV? (b) Prior to this event how would you rate your understanding of cervical cancer? (c) After this event how would you rate your understanding of HPV/cervical cancer? An average score was used.

\section{Actual Knowledge}

Knowledge of HPV and cervical cancer were assessed preand post-forums using a five-item dichotomous quiz (yes or no response options). The pre- and post-assessment questions (actual knowledge) included: two questions on HPV, two questions on cervical cancer, and one question related to the HPV vaccine. Actual knowledge was quantified by the number of correct answers out of five pre- and post-assessment questions. Pretest assessment forum questions were: (a) Is HPV the most commonly diagnosed sexually transmitted disease? (b) If you have HPV, you will develop cervical cancer (c) Cervical cancer is only caused by two forms of HPV? (d) The HPV vaccine will only work if you take it before you become an adult (e) The HPV vaccine is about 98-99\% effective. Posttest questions were: (a) HPV is a bacteria and there are no known cures for HPV? (b) HPV is the cause of the overwhelming majority of cervical cancer cases (c) Can cervical cancer be caused by all forms of HPV? (d) It is best to take the HPV vaccine before becoming a teenager (e) The HPV vaccine is about $60 \%$ effective. An average score was used for all analyses. To minimize our participants' familiarity with the pre/post assessments, we used different items that measured similar knowledge constructs.

\section{Healthcare}

We used four questions to assess health practices and attitudes: (a) Do you have a regular doctor? Response options were yes or no. (b) Do you have health insurance? Response options were yes or no. (c) How trusting are you of clinical trials? Response options ranged from not trusting at all to very trusting. (d) How trusting are you of vaccines? Response options ranged from not trusting at all to very trusting.

\section{Data Analysis}

Descriptive statistics were used to assess all variables. The actual knowledge variable was quantified by using the number of correct answers out of five questions. Paired t-tests 
were used to determine whether scores within groups were significantly different prior/after and pre/post forum activities for all groups. Generalized linear regressions were run to assess whether associations enhanced the effect of differences between perceived scores. Statistically significant differences are reported for $p$ values that are less than 0.05 (at the $95 \%$ confidence level).

\section{Results}

\section{Participants}

From an overall sample of over 500 participants, 412 questionnaires $(82.6 \%)$ were collected. Descriptive statistics for the population at the forums are presented in Table 1. Participants were predominantly African American (86.2\%), females (90.4\%) between 20 and 30 years of age (37.3\%), who were somewhat religious $(52.1 \%)$. Since the forums were held on college campuses, several nursing program professors at both schools encouraged their students to attend the forums. As a result, many of the participants were students in the nursing programs at Kennedy-King and Richard M. Daley Colleges.

\section{Effectiveness of Oral Presentations}

The pharmaceutical industry representative had the highest efficacy rating compared to the other forum presenters. The oral presentation ratings were as follows: academic/

Table 1 Study demographic frequencies

\begin{tabular}{|c|c|c|c|c|c|c|c|}
\hline Variable & Total & $\begin{array}{l}\text { African } \\
\text { American }\end{array}$ & $\begin{array}{l}\text { Hispanic } \\
\text { American }\end{array}$ & $\begin{array}{l}\text { European } \\
\text { American }\end{array}$ & Asian American & Other/multiple & p-value \\
\hline $\mathrm{N}$ & 412 & 355 & 29 & 9 & 6 & 13 & $>0.001$ \\
\hline \multicolumn{8}{|l|}{ Site } \\
\hline $\mathrm{KKC}$ & - & 85.1 & 7.7 & 2.0 & 1.6 & 3.6 & \\
\hline Daley & - & 54.5 & 23.2 & 9.3 & 4.6 & 9.3 & $>0.001$ \\
\hline$\%$ female & 90.4 & 90.9 & 86.2 & 78 & 83 & 100 & 0.55 \\
\hline Have health insurance & 81.3 & 81.6 & 62.1 & 88.9 & 83.3 & 85.6 & 0.09 \\
\hline Have primary physician & 81.3 & 81.6 & 62.1 & 88.9 & 83.3 & 85.6 & 0.09 \\
\hline \multicolumn{8}{|l|}{ Age } \\
\hline$<20$ & 7.5 & 6.9 & 0 & 11.1 & 0 & 38.5 & \\
\hline $20-30$ & 37.3 & 33.3 & 82.7 & 33.3 & 50 & 15.4 & \\
\hline $31-40$ & 30.5 & 33.0 & 12.3 & 33.3 & 16.7 & 15.4 & \\
\hline $41-50$ & 15.1 & 16.0 & 0 & 22.2 & 33.3 & 23.1 & \\
\hline $51-60$ & 8.6 & 9.9 & 0 & 0 & 0 & 7.7 & \\
\hline $61+$ & 1.0 & 0.4 & 0 & 0 & 0 & 0 & 0.02 \\
\hline \multicolumn{8}{|l|}{ Income } \\
\hline$<\$ 10,000$ & 21.3 & 24.4 & 4.2 & 12.5 & 0 & 20.0 & \\
\hline$\$ 10-\$ 30 \mathrm{k}$ & 31.5 & 30.2 & 41.2 & 50.0 & 16.7 & 30.0 & \\
\hline$\$ 30-\$ 50 \mathrm{k}$ & 16.9 & 15.1 & 20.1 & 0 & 66.7 & 20.0 & \\
\hline$\$ 50-\$ 70 \mathrm{k}$ & 12.6 & 11.8 & 25.0 & 12.5 & 0 & 10.0 & \\
\hline$\$ 70-\$ 90 \mathrm{k}$ & 7.1 & 6.8 & 4.2 & 25.0 & 0 & 10.0 & \\
\hline$\$ 90 \mathrm{k}+$ & 10.6 & 11.8 & 4.2 & 0 & 16.7 & 10.0 & 0.86 \\
\hline \multicolumn{8}{|l|}{ Education } \\
\hline Vocation & 12.9 & 12.7 & 13.8 & 0 & 16.7 & 10.0 & \\
\hline H.S. grad & 30.1 & 31.7 & 34.5 & 55.6 & 16.7 & 20.0 & \\
\hline Associate & 34.4 & 35.3 & 41.4 & 33.3 & 16.7 & 60.0 & \\
\hline Bachelor & 12.2 & 11.8 & 10.4 & 11.1 & 50.0 & 10.0 & \\
\hline Masters & 6.1 & 7.7 & 0 & 0 & 0 & 0 & \\
\hline Professional & 0.7 & 0.9 & 0 & 0 & 0 & 0 & 0.39 \\
\hline \multicolumn{8}{|l|}{ Religiosity } \\
\hline Very religious & 41.3 & 46.5 & 13.8 & 11.1 & 66.7 & 16.7 & \\
\hline Somewhat & 52.1 & 46.5 & 86.2 & 66.7 & 16.7 & 83.3 & \\
\hline Not religious & 6.6 & 7.0 & 0 & 22.2 & 16.7 & 0 & 0.03 \\
\hline
\end{tabular}


pharmaceutical consultant $(71.9 \%)$, researcher $(60.8 \%)$, gynecologist (38.5\%), public health administrator (37.3\%), academician $(32.7 \%)$, and survivor $(29.1 \%)$. The percent difference between the various speakers compared to the pharmaceutical industry representative was $11.1 \%, 33.4 \%$, $34.6,39.2$, and $42.8 \%$, respectively.

\section{HPV and Cervical Cancer Knowledge}

Table 2 shows mean scores based on percentage increase for perceived and actual knowledge of HPV and cervical cancer by race/ethnicity. Perceived knowledge of HPV and cervical cancer before and after the forum was only significantly different for African Americans, $t(354)=5.66$, $\mathrm{p}=<0.001$. Actual pre- and post-forum knowledge was significantly different for African American, t (265) $=-6.14$, $\mathrm{p}=<0.001$ and Hispanic American $\mathrm{t}(28)=-2.17, \mathrm{p}=0.039$ participants.

\section{Associations of Perceived Knowledge for African Americans}

Table 3 shows the most significant associations from the questionnaire for African Americans. For perceived knowledge prior to the forums, the significant associations were health insurance status $(\beta=0.814, p=0.010)$, trust in clinical trials $(\beta=0.200, p=0.005)$, and gender $(\beta=0.168$, $p=0.018$ ). For perceived knowledge after the event, the significant associations of the mean score were having a regular doctor $(\beta=2.15, p=0.033)$ and trust of vaccines $(\beta=2.06, p=0.041)$. Having health insurance and a regular doctor correlated with higher scores. For trust associations, the higher the trust ratings of clinical trials and vaccinations, the higher the pre- and post-forum knowledge mean scores. For pre-assessment mean knowledge scores, the main significant association was age $(\beta=-0.262, p=0.028)$. For mean post-forum knowledge scores the main significant association was also age $(\beta=-0.364, p=0.002)$.

\section{Discussion}

Cervical cancer is a preventable and treatable disease that continues to negatively impact African American communities [1]. Screening has played a critical role in reducing HPV-related cancer mortality among African American women $[4,35]$. In countries with established and effective HPV vaccination programs, there are substantial declines in HPV precancerous outcomes (i.e. CIN2/3) [36]. Unfortunately, HPV vaccine acceptance in minority populations lags behind that of Whites [5]. While the causes of HPV immunization disparities between African Americans and their Hispanic and White counterparts remain unclear, communities continue to use forums to improve cancer education $[27,29,30]$. Community forums have emerged as an effective education strategy because they are grounded in community-based participatory research principles that invite

Table 2 Mean scores for perceived and real knowledge of HPV and cervical cancer

\begin{tabular}{llllll}
\hline & African American & Hispanic American & European American & Asian American & Other/multiple \\
\hline $\begin{array}{l}\text { Perceived scores } \\
\quad\end{array}$ & & & & & \\
Prior & $3.31(1.12)$ & $2.96(1.07)$ & $3.39(1.14)$ & $4.70(1.10)$ & $3.58(0.70)$ \\
After & $4.45(0.68)$ & $4.50(0.51)$ & $4.71(0.49)$ & & $4.67(0.52)$ \\
Real scores & & & & N/A & $3.37(0.53)$ \\
Pre test & $2.94(1.01)$ & $3.00(1.00)$ & $3.25(0.96)$ & N/A & $4.33(1.15)$ \\
Post test & $4.08(0.96)$ & $4.22(0.83)$ & $4.25(0.96)$ & $0.58)$ \\
\hline
\end{tabular}

Mean scores (standard deviation)

Table 3 Associations of perceived knowledge in African Americans

\begin{tabular}{lllllll}
\hline & Gender & Age & Insurance & Physician & Trust in clinical trials & Trust in vaccines \\
\hline $\begin{array}{l}\text { Perceived scores } \\
\text { Prior }\end{array}$ & $0.168(0.005)$ & NS & & & & \\
After & NS & NS & NS & NS & $0.200(0.005)$ & NS \\
$\begin{array}{l}\text { Real scores } \\
\text { Pretest }\end{array}$ & NS & $-0.262(0.02)$ & NS & NS & NS & NS \\
Posttest & NS & $-0.364(0.002)$ & NS & NS & NS & NS \\
\hline
\end{tabular}

Beta and p-values in parentheses for significant associations of perceived and real knowledge of HPV and cervical cancer in African Americans 
communities to be partners in identifying health challenges and crafting strategies to improve health outcomes [27, 30].

The findings in this study support the effectiveness of community forums. The data show that, for African Americans in attendance, perceived knowledge increased significantly after the forums. Perceived knowledge prior to the forums varied with gender, access to care (health insurance status), and trust in clinical trials. After the forum, increased perceived knowledge was associated with having health insurance and having trust in vaccines. The persistent association before and after the forums between trust and perceived knowledge is not surprising. African Americans mistrust of medical research is a significant barrier to their participation in clinical studies [37]. This mistrust may be due in part to the historical unethical misuse of Black bodies for research advancements as exemplified by the Tuskegee Syphilis Study and the case of Henrietta Lacks [38]. In our study, the positive relationship between trust in vaccines and perceived knowledge that was realized after the forums underscores the usefulness of this mode of information sharing for cancer prevention education and trust building.

While the scope of our study did not allow for empirical analysis of the relationship between message mode and perceived and actual knowledge, it is plausible the composition (e.g. primarily Black and Hispanic representation) and structure of the panel contributed to message acceptability and reduced suspicion. The panel was composed of ethnically diverse scientists, community leaders, physicians, a cancer survivor, and an academician/pharmaceutical consultant, all of whom encouraged immunization of young women in accordance with recommendations from the Advisory Committee on Immunization Practices. Some members of the panel also shared personal narratives about their children's and their own experiences with the vaccine; including minor side effects such as soreness at the vaccination site that eventually subsided. In addition, the forum opened with a presentation about the historical context and acknowledgement of minority communities' mistrust of medical research. This overall approach may help to explain the shift in significant association after the forum from trust in clinical trials to trust in vaccines. The inclusion of a pharmaceutical consultant for one company that markets one of the US Food and Drug Administration approved HPV vaccines may have engendered trust, as well. The presentation from the academician/pharmaceutical consultant included description of the methodology used to develop and test the vaccine and assurance of its safety and efficacy. Post forum evaluations were very favorable for the academician/pharmaceutical consultant, with participants rating him two to three times higher than other panelists. The engagement of the pharmaceutical industry through a consultant gave participants a chance to interrogate the vaccine development process and this may have minimized their mistrust.
The precise contribution the pharmaceutical industry can make to public health education and cervical cancer prevention warrants further inquiry. The pharmaceutical industry has been criticized for market driven profiteering that is not necessarily in the interest of consumers [39]. As a result, their engagement in health issues is widely viewed with suspicion [40]. In this study, however, the inclusion of a pharmaceutical representative appeared to increase vaccine acceptance. Future work to explore this possible benefit will need to identify the precise advantages and best practices for engaging the pharmaceutical industry. Researchers will also need to untangle the complexities surrounding conflict of interest, ethics, fair trade, and population safety and interest.

Whereas perceived knowledge of HPV and cervical cancer was explained by access to care and trust in clinical trials/vaccines, actual knowledge was significantly explained by age at pre- and post-assessment. Older adults in our sample were less knowledgeable about HPV and cervical cancer than younger participants. This finding mirrors other studies that have also reported age differences in HPV and HPV-related cancer knowledge [10, 11]. Maness and Reitzel, for example, reported increasing deficits in knowledge in a sample ranging in age from 18 to over 60 years old [10]. Seventy-seven percent of participants in the 18-39 age group knew of the association between HPV and cervical cancer, compared to $47 \%$ of participants in the 60 and above age group. Although participants in our study were relatively young with majority (37.3) being $20-30$ years-old, the agerelated variations in knowledge were still evident. This early decline in knowledge that continues across increasing age underscores the need to tailor forums and other intervention programs to reach diverse audiences with varying knowledge needs. The primary focus of HPV vaccination is adolescents, but parents and grandparents or other caregivers are critical in ultimate vaccination decisions [41]. The success of HPVrelated education campaigns, therefore, rests on educating the general population about the association between HPV and cancer.

\section{Strengths and Limitations}

This study adds to the growing body of literature that identifies health forums as a useful medium for community education [25-27, 29]. It also provides preliminary evidence of a format and panel composition that might be very effective in building trust and increasing HPV knowledge and vaccine acceptance in African American communities. Patterns of social interactions in these communities are oriented toward communal decision making and collective action [27, 32]. Therefore, the forums, which brought together a wide cross-section of stakeholders and used history and communal experiences to 
contextualize dialogue, were culturally congruent and responsive to community values and concerns. This intervention approach holds promise as a model than can be used to help realize American Cancer Society's mission to achieve $80 \%$ preteen HPV vaccination rate by 2026 and reduce cervical cancer disparities.

While this study expands understanding of the utility of forums in cancer prevention education, the findings should be interpreted within the limitations of the work. First, the forums were convened on college campuses; as a result, the sample was comprised of a disproportionately high number of female nursing students. The sample therefore was not representative of the general population. Second, we did not use a comparison group; so, while we can assess overall changes in perceived knowledge, the scope of this work does not permit definitive conclusions about the efficacy of this strategy compared to other cancer education approaches. Third, the study design cannot prove causation; and fourth and we did not explore the relationship between perceived and actual knowledge and cancer prevention behaviors. Future studies could consider using a randomized control trial design and convening forums in a variety of community settings to attract a more representative audience.

\section{Conclusion}

Most cases of cervical cancer can be prevented by HPV vaccination [35]. Therefore, the reduction of cervical cancer disparities among African Americans hinges on increasing HPV vaccination uptake in this population. Community health forums facilitate community partnership and dialogue; so, they have the potential to reduce mistrust and increase knowledge and possibly vaccine uptake. Forums that respond to the cultural and historical context and include a broad representation of stakeholders may be particularly effective. The model presented in this study is a potentially useful strategy to increase HPV and cervical cancer knowledge in African American communities. Additional research is needed to clarify how different elements of the model contribute to overall education intervention success.

Funding This study was funded with developmental funds from the University of Chicago Cancer Center.

\section{Compliance with Ethical Standards}

Conflict of interest Dr. Kenneth Alexander is a consultant with Merck Pharmaceuticals and received a speaker honorarium. All remaining authors declare that they have no conflict of interest.

\section{References}

1. Centers for Disease Control and Prevention. (2016). The link between HPV and cancer. Retrieved from https://www.cdc.gov/ hpv/parents/cancer.html. Accessed 16 Nov 2017.

2. Aref-Adib, M., \& Freeman-Wang, T. (2016). Cervical cancer prevention and screening: The role of human papillomavirus testing. The Obstetrician \& Gynaecologist, 18(4), 251-263.

3. American Cancer Society. (2018). Cancer facts \& figures 2018. Atlanta: American Cancer Society.

4. American Cancer Society. (2016). The American Cancer Society guidelines for the prevention and early detection of cervical cancer. Retrieved from https://www.cancer.org/cancer/cervical-cance $\mathrm{r} /$ prevention-and-early-detection/cervical-cancer-screening-guide lines.html. Accessed 16 Nov 2017.

5. Centers for Disease Control and Prevention. (2017). Cervical cancer rates by race and ethnicity. Retrieved from https://www.cdc. gov/cancer/cervical/statistics/race.htm. Accessed 16 Nov 2017

6. Howell, E. A., Chen, Y. T., \& Concato, J. (1999). Differences in cervical cancer mortality among black and white women. Obstetrics and Gynecology, 94(4), 509-515.

7. Patel, D. A., et al. (2005). A population-based study of racial and ethnic differences in survival among women with invasive cervical cancer: Analysis of surveillance, epidemiology, and end results data. Gynecologic Oncology, 97(2), 550-558.

8. Movva, S., et al. (2008). Racial differences in cervical cancer survival in the Detroit metropolitan area. Cancer, 112(6), 1264-1271.

9. Brookfield, K. F., et al. (2009). Disparities in survival among women with invasive cervical cancer: A problem of access to care. Cancer, 115(1), 166-178.

10. Maness, S. B., et al. (2016). HPV awareness, knowledge and vaccination attitudes among church-going African-American women. American Journal of Health Behavior, 40(6), 771-778.

11. McBride, K. R., \& Singh, S. (2018). Predictors of adults' knowledge and awareness of HPV, HPV-associated cancers, and the HPV vaccine: Implications for health education. Health Education \& Behavior, 45(1), 68-76.

12. Perkins, R. B., et al. (2015). Effectiveness of a provider-focused intervention to improve HPV vaccination rates in boys and girls. Vaccine, 33(9), 1223-1229.

13. Smulian, E. A., Mitchell, K. R., \& Stokley, S. (2016). Interventions to increase HPV vaccination coverage: A systematic review. Human Vaccines \& Immunotherapeutics, 12(6), 1566-1588.

14. Walling, E. B., et al. (2016). Interventions to improve HPV vaccine uptake: A systematic review. Pediatrics, 138(1), e20153863.

15. Perkins, R. B., et al. (2014). Missed opportunities for HPV vaccination in adolescent girls: A qualitative study. Pediatrics, 134, e666-e674.

16. Joseph, N. P., et al. (2014). Racial and ethnic differences in HPV knowledge, attitudes, and vaccination rates among low-income African-American, Haitian, Latina, and Caucasian young adult women. Journal of Pediatric and Adolescent Gynecology, 27(2), 83-92.

17. Katz, I. T., et al. (2016). Barriers to HPV immunization among blacks and latinos: A qualitative analysis of caregivers, adolescents, and providers. BMC Public Health, 16(1), 874.

18. Martin, J. Y., et al. (2017). Racial disparities in the utilization of preventive health services among older women with early-stage endometrial cancer enrolled in Medicare. Cancer Medicine, 6(9), 2153-2163.

19. Gilkey, M. B., \& McRee, A.-L. (2016). Provider communication about HPV vaccination: A systematic review. Human Vaccines \& Immunotherapeutics, 12(6), 1454-1468.

20. Centers for Disease Control and Prevention. (2017). Prevalence of HPV in adults aged 18-69: United States, 2011-2014. Retrieved 
December 12, 2018 from https://www.cdc.gov/nchs/products/ databriefs/db280.htm. Accessed 16 Nov 2017.

21. Fu, L. Y., et al. (2014). Educational interventions to increase HPV vaccination acceptance: A systematic review. Vaccine, 32(17), 1901-1920.

22. Niccolai, L. M., \& Hansen, C. E. (2015). Practice-and community-based interventions to increase human papillomavirus vaccine coverage: A systematic review. JAMA Pediatrics, 169(7), 686-692.

23. Iwelunmor, J., Newsome, V., \& Airhihenbuwa, C. O. (2014). Framing the impact of culture on health: A systematic review of the PEN-3 cultural model and its application in public health research and interventions. Ethnicity \& Health, 19(1), 20-46.

24. Betsch, C., et al. (2016). Improving medical decision making and health promotion through culture-sensitive health communication: An agenda for science and practice. Medical Decision Making, 36(7), 811-833.

25. Bharmal, N., et al. (2016). A community engagement symposium to prevent and improve stroke outcomes in diverse communities. Progress in Community Health Partnerships, 10(1), 149-158.

26. Davis, J. L., et al. (2012). An innovative approach for community engagement: Using an audience response system. Journal of Health Disparities Research and Practice, 5(2), 1.

27. Jimenez, J., et al. (2018). Community engagement for identifying cancer education needs in Puerto Rico. Journal of Cancer Education, 33, 12-20.

28. Jonassaint, C. R., et al. (2010). Regional differences in awareness and attitudes regarding genetic testing for disease risk and ancestry. Human Genetics, 128(3), 249-260.

29. Bullock, K., \& McGraw, S. A. (2006). A community capacityenhancement approach to breast and cervical cancer screening among older women of color. Health and Social Work, 31(1), $16-25$.

30. Meade, C. D., et al. (2007). Impacting health disparities through community outreach: Utilizing the CLEAN look (culture, literacy, education, assessment, and networking). Cancer Control, 14(1), $70-77$.
31. Maertens, J. A., et al. (2017). Using community engagement to develop a web-based intervention for latinos about the HPV vaccine. Journal of Health Communication, 22(4), 285-293.

32. Lincoln, C. E., \& Mamiya, L. H. (1990). The black church in the African American experience. Durham: Duke University Press.

33. Mello, M. M., Abiola, S., \& Colgrove, J. (2012). Pharmaceutical companies' role in state vaccination policymaking: The case of human papillomavirus vaccination. American Journal of Public Health, 102(5), 893-898.

34. Illinois Department of Public Health. (2006). Cervical cancer task force annual report. Chicago, Illinois.

35. Dochez, C., et al. (2014). HPV vaccines to prevent cervical cancer and genital warts: An update. Vaccine, 32(14), 1595-1601.

36. Sankaranarayanan, R. (2015). HPV vaccination: The most pragmatic cervical cancer primary prevention strategy. International Journal of Gynecology \& Obstetrics, 131, S33-S35.

37. Crawley, L. M. (2001). African-American participation in clinical trials: Situating trust and trustworthiness. Journal of the National Medical Association, 93(12 Suppl), 14S-17S.

38. Scharff, D. P., et al. (2010). More than Tuskegee: Understanding mistrust about research participation. Journal of Health Care for the Poor and Underserved, 21(3), 879-897.

39. Angell, M. (2004). Excess in the pharmaceutical industry. CMAJ: Canadian Medical Association Journal, 171(12), 1451-1453.

40. Grande, D., Shea, J. A., \& Armstrong, K. (2012). Pharmaceutical industry gifts to physicians: Patient beliefs and trust in physicians and the health care system. Journal of General Internal Medicine, 27(3), 274-279.

41. Holman, D. M., et al. (2014). Barriers to human papillomavirus vaccination among US adolescents: A systematic review of the literature. JAMA Pediatrics, 168(1), 76-82.

Publisher's Note Springer Nature remains neutral with regard to jurisdictional claims in published maps and institutional affiliations. 